\title{
Spectroscopic observations of ices around embedded young stellar objects in the Large Magellanic Cloud with AKARI
}

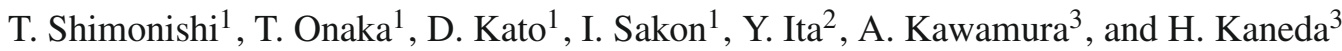 \\ 1 Department of Astronomy, Graduate School of Science, The University of Tokyo, 7-3-1 Hongo, Bunkyo-ku, \\ Tokyo 113-0033, Japan \\ e-mail: shimonishi@astron.s.u-tokyo.ac.jp \\ 2 National Astronomical Observatory of Japan, 2-21-1 Osawa, Mitaka, Tokyo, 181-8588, Japan \\ 3 Graduate School of Science, Nagoya University, Chikusa-ku, Nagoya, 464-8602, Japan
}

Received 7 December 2009 / Accepted 12 March 2010

\section{ABSTRACT}

\begin{abstract}
Aims. The aim of this study is to understand the chemical conditions of ices around embedded young stellar objects (YSOs) in the metal-poor Large Magellanic Cloud (LMC).

Methods. We performed near-infrared $(2.5-5 \mu \mathrm{m})$ spectroscopic observations toward 12 massive embedded YSOs and their candidates in the LMC using the infrared camera (IRC) onboard AKARI. We estimated the column densities of the $\mathrm{H}_{2} \mathrm{O}, \mathrm{CO}_{2}$, and $\mathrm{CO}_{\text {ices }}$ based on their 3.05, 4.27, and $4.67 \mu \mathrm{m}$ absorption features, and we investigated the correlation between ice abundances and physical properties of YSOs.

Results. The ice absorption features of $\mathrm{H}_{2} \mathrm{O}, \mathrm{CO}_{2},{ }^{13} \mathrm{CO}_{2}, \mathrm{CO}, \mathrm{CH}_{3} \mathrm{OH}$, and possibly XCN are detected in the spectra. In addition, hydrogen recombination lines and PAH emission bands are detected toward the majority of the targets. The derived typical $\mathrm{CO}_{2} / \mathrm{H}_{2} \mathrm{O}$ ice ratio of our samples $(\sim 0.36 \pm 0.09)$ is greater than that of Galactic massive YSOs $(\sim 0.17 \pm 0.03)$, while the $\mathrm{CO} / \mathrm{H}_{2} \mathrm{O}$ ice ratio is comparable. It is shown that the $\mathrm{CO}_{2}$ ice abundance does not correlate with the observed characteristics of YSOs: the strength of hydrogen recombination line and the total luminosity. Likewise, clear correlation is not seen between the CO ice abundance and YSO characteristics, but it is suggested that the CO ice abundance of luminous samples is significantly lower than in other samples. Conclusions. The systematic difference in the $\mathrm{CO} 2$ ice abundance around the LMC's massive YSOs, which was suggested by previous studies, is confirmed with the new near-IR data. We suggest that the strong ultraviolet radiation field and/or the high dust temperature in the $\mathrm{LMC}$ are responsible for the observed high abundance of the $\mathrm{CO}_{2}$ ice. It is suggested that the internal stellar radiation does not play an important role in the evolution of the $\mathrm{CO}_{2}$ ice around a massive YSO, while more volatile molecules like $\mathrm{CO}$ are susceptible to the effect of the stellar radiation.
\end{abstract}

Key words. astrochemistry - circumstellar matter - ISM: abundances - ISM: molecules - Magellanic Clouds - infrared: ISM

\section{Introduction}

The infrared spectra of embedded young stellar objects (YSOs) show absorption features originating in various ice species (solid state molecules, e.g., Gerakines et al. 1999; Gibb et al. 2004; Boogert et al. 2008). It is believed that a large amount of heavy elements and complex molecules of the interstellar medium (ISM) are preserved as ices in the dense and cold regions $\left(n_{\mathrm{H}} \geq 10^{4} \mathrm{~cm}^{-3}, T \sim 10-20 \mathrm{~K}\right)$, such as an envelope of a deeply embedded YSO (van Dishoeck \& Blake 1998; Boogert $\&$ Ehrenfreund 2004). Since the star formation proceeds in the region of dense molecular clouds, these ices dominate the chemical evolution of YSOs. Absorption profiles of ices are known to be sensitive to a chemical composition and a temperature of dust grains, and ices are important tracers of the thermal history of circumstellar environments of YSOs (e.g., Pontoppidan et al. 2008; Zasowski et al. 2009). Thus, investigating the compositions of ices as a function of physical environments is crucial for understanding the chemical evolution of YSOs and is one of the key topics in the current astrochemistry. Ices are also detected toward solar system objects such as comets, icy satellites, and Mars. Interstellar ices are thought to be taken into planets and comets as a result of subsequent planetary formation activities (Ehrenfreund \& Schutte 2000). Therefore the chemical evolution of ices around YSOs is also of interest in terms of chemical conditions of the planetary formation.

Observations of ices around extragalactic YSOs are one of the challenges in current ice studies (Shimonishi et al. 2008; Oliveira et al. 2009; Seale et al. 2009; van Loon et al. 2005, 2010a). So far, infrared spectroscopic observations of extragalactic embedded YSOs are still limited by observational difficulties, and their circumstellar chemistry is poorly understood. However, it is probable that different galactic environments (e.g., metallicity or radiation field) could affect the properties of circumstellar material. Thus detailed investigations of extragalactic YSOs provide us important information for understanding the diversity of chemical conditions of YSOs in the universe. Thanks to the progress of infrared space telescopes, such as AKARI (Murakami et al. 2007) and Spitzer (Werner et al. 2004), we are now able to extend the study of ices around YSOs to extragalactic objects.

The Large Magellanic Cloud (LMC), the nearest irregular galaxy to our Galaxy, offers an ideal environment for studying extragalactic star formations since individual YSOs can be identified with reasonable spatial resolution $\left(1^{\prime \prime} \sim 0.25 \mathrm{pc}\right)$ thanks to its proximity ( $\sim 50 \mathrm{kpc}$; Alves 2004$)$. A unique metalpoor environment of the LMC $\left(Z \sim 0.3 Z_{\odot}\right.$, Luck et al. 1998) 
results in a generally high UV radiation field over the galaxy, and these factors can affect the chemical conditions of circumstellar materials. The nearly face-on viewing angle of this galaxy ( 35 degrees, van der Marel \& Cioni 2001) allows correlation studies of individual YSOs and the ISM. Because of these advantages, various types of surveys have been performed toward the LMC, which provide information of both ISM and stars over a wide wavelength range (e.g., Zaritsky et al. 2004; Meixner et al. 2006; Kato et al. 2007).

Shimonishi et al. (2008) spectroscopically confirmed seven massive YSOs in the LMC based on the AKARI LMC spectroscopic survey (Ita et al. 2008) and reported the detection of the $\mathrm{H}_{2} \mathrm{O}$ and $\mathrm{CO}_{2}$ ices toward these objects. Oliveira et al. (2009) analyzed the $15.2 \mu \mathrm{m}$ feature of the $\mathrm{CO}_{2}$ ice for 15 embedded YSOs in the LMC and discuss the chemical properties of the ice mantles. The $\mathrm{H}_{2} \mathrm{O}$ and $\mathrm{CO}_{2}$ ices are ubiquitous and are the major components of interstellar ices. Based on the low-resolution $(R \sim 20)$ spectra obtained in Shimonishi et al. (2008), we derived a typical $\mathrm{CO}_{2} / \mathrm{H}_{2} \mathrm{O}$ ice ratio and showed that the ratio around the LMC's massive YSOs $(0.45 \pm 0.17)$ is higher than previously reported toward Galactic massive YSOs $(0.17 \pm 0.03$, Gerakines et al. 1999). The result suggests the chemically different nature of YSOs in the metal-poor galaxy.

An accurate determination of column densities of major ice species is crucial when discussing the variation of ice abundances between the objects. However, the uncertainties in the derived column densities from our previous low-resolution spectra are large, and the number of samples for which the column densities of major ice species are determined is still small. In addition, the features of relatively minor ice species, such as $\mathrm{CH}_{3} \mathrm{OH}$ and $\mathrm{CO}$ are difficult to identify by the low-resolution spectra. Investigation of these minor ices should help in constraining the processing of ices in a YSO envelope (e.g., Dartois et al. 1999; Thi et al. 2006). Thus follow-up observations with higher spectral resolution are required to further understand the chemical conditions of extragalactic YSOs.

In the present study, we used the higher spectral resolution $(R \sim 80)$ mode of the infrared camera (IRC, Onaka et al. 2007) onboard AKARI to observe massive embedded YSOs and their candidates in the LMC. The correlation between the chemical properties of ices and the YSO characteristics, which could not be discussed in Shimonishi et al. (2008) due to the large uncertainties of the observed spectra, is discussed in this paper. In addition, the improved calculation method of the ice column density by using the curve-of-growth is presented in an Appendix.

\section{Targets}

The sample of massive YSOs presented in this study is mainly selected from Shimonishi et al. (2008), which spectroscopically discovered several massive YSOs in the LMC by using a combination of the low-resolution, near-infrared spectroscopic survey and the color-color diagram. The $\mathrm{H}_{2} \mathrm{O}$ and $\mathrm{CO}_{2}$ ice features toward these targets have already been reported in Shimonishi et al. (2008), and this indicates the embedded nature of these YSOs. In addition, we include several YSO "candidates". These objects were selected by the same photometric criteria described in Shimonishi et al. (2008) and their infrared spectral energy distributions (SEDs) are red enough to be identified as a YSO. But absorption features of ices seen in their low-resolution 2-5 $\mu \mathrm{m}$ spectra are too weak to definitely classify them as YSOs, so they are not included in the YSO list of Shimonishi et al. (2008). Some other targets were selected based on the YSO candidates catalog presented in Whitney et al. (2008), which performed photometric selection of YSOs using the database of Spitzer SAGE project (Meixner et al. 2006). We selected relatively bright and red objects from their catalog. van Loon et al. (2005) observed IRAS 05328-6827 with the infrared spectrograph (IRS, Houck et al. 2004) onboard Spitzer and the infrared spectrometer and array camera (ISAAC) at the Very Large Telescope (VLT), and report the presence of ice features in its near- to mid-infrared spectrum. This object is also added to the present targets.

To understand the nature of the targets, we carried out the SED fitting of each YSO by using the Online SED Fitter ${ }^{1}$ (Robitaille et al. 2007). The photometric dataset used for the SED fitting are obtained from the available database and recent YSO catalogs (Zaritsky et al. 2004; Meixner et al. 2006; Kato et al. 2007; Ita et al. 2008; Gruendl \& Chu 2009), and 2-24 $\mu \mathrm{m}$ data were typically used as the input data. The estimated total luminosities and masses of our targets are $\sim 4-340 \times 10^{3} L_{\odot}$ and 12-47 $M_{\odot}$, respectively. Thus the targets in the present study are massive YSOs. Details of all the targets are listed in Table 1.

\section{Observation and data reduction}

Spectra of $2-5 \mu \mathrm{m}$ presented in this paper were obtained as a part of AKARI post-helium open time program "ices around extragalactic young stellar objects" (IEYSO, PI: Shimonishi) and AKARI director's time (DT) observations. AKARI is the first Japanese satellite dedicated to infrared astronomy launched in 2006. The telescope system and the scientific instruments onboard were cooled down by the liquid helium with mechanical coolers. The AKARI cryogen boiled off on 2007 August 26, 550 days after launch. All of the data presented here were obtained after the exhaustion of liquid helium.

The slit-less NG spectroscopy mode with the $\mathrm{Np}$ point source aperture was used to obtain spectra between 2.5 and $5 \mu \mathrm{m}$ with a spectral resolution of $R \sim 80$. The wavelength accuracy of the spectroscopy is estimated to be $\sim 0.01-0.015 \mu \mathrm{m}$. The spatial resolution of a point source is approximately 5-8 arcsec. Overlapping of dispersed spectra with other objects is a serious problem for slit-less spectroscopies, which makes extraction of reliable spectra difficult. The use of the $\mathrm{Np}$ point source aperture reduces the effect of spectral overlapping and enables spectra to be extracted from the objects located in crowded regions (see Ohyama et al. 2007, and AKARI data users manual for details of the IRC spectroscopy).

The basic spectral analysis was performed using the standard IDL pipeline prepared for reducing AKARI IRC post-helium mission data. Raw data were converted to dark-subtracted and linearity-corrected frames in the pipeline. We used a newly calibrated spectral response curve to analyze the present spectra. At that time, appropriate flat field data were not available for post-helium Np data. However, because of the faintness of the natural background and the small pixel-to-pixel variation of the flux at the Np aperture, there are no significant differences between the spectra with and without flat-fielding (AKARI data users manual ver. 1.3). In fact, spectra processed without the flat-fielding is better in terms of $\mathrm{S} / \mathrm{N}$ because of the relatively low $\mathrm{S} / \mathrm{N}$ of the flat field data. Therefore, we did not perform the flat-fielding in the pipeline. Four to seven pixels were integrated in the spatial direction to extract the point source spectrum. Each pixel was smoothed in the dispersion direction across the width of three pixels to suppress the effect of bad pixels. Background signals of each source were estimated from the signal counts of the adjacent region of the target spectrum and subtracted.

\footnotetext{
1 The Online SED Fitter is available at http: //caravan . astro.wisc. edu/protostars/sedfitter.php
} 
Table 1. Targets of the present observations.

\begin{tabular}{lcccccl}
\hline \hline Number & ID & Obs. ID & Obs. Date & RA[J2000] & Dec[J2000] & Reference \\
\hline ST1 & IRAS 05400-7013 & 5126560 & 2008 Apr. 12 & $05: 39: 31.15$ & $-70: 12: 16.8$ & 1,2 \\
ST2 & NGC 1936 & 5126562 & 2008 May 25 & $05: 22: 12.56$ & $-67: 58: 32.2$ & 1 \\
ST3 & $\ldots$ & 3410003 & 2008 Dec. 11 & $05: 25: 46.69$ & $-66: 14: 11.3$ & 1,3 \\
ST4 & IRAS F05148-6715 & 3410004 & 2008 Dec. 27 & $05: 14: 49.41$ & $-67: 12: 21.5$ & 1,3 \\
ST5 & IRAS 05311-6836 & 5126561 & 2008 May 11 & $05: 30: 54.27$ & $-68: 34: 28.2$ & 1,2 \\
ST6 & 05394112-6929166 & 5126563 & 2008 Apr. 19 & $05: 39: 41.08$ & $-69: 29: 16.8$ & 1 \\
ST7 & IRAS 05240-6809 & 5126567 & 2008 May 23 & $05: 23: 51.15$ & $-68: 07: 12.2$ & 1,3 \\
ST8 & MSX LMC 1786 & 5126573 & 2008 May 2 & $05: 37: 28.17$ & $-69: 08: 47.0$ & $3,4,5,6$ \\
ST9 & IRAS 05237-6755 & 5126568 & 2008 May 26 & $05: 23: 35.53$ & $-67: 52: 35.5$ & 2,4 \\
ST10 & MSX LMC 1229 & 5200384 & 2008 Dec. 21 & $04: 56: 40.80$ & $-66: 32: 30.4$ & $2,4,5$ \\
ST11 & IRAS05270-6851 & 5126571 & 2008 May 11 & $05: 26: 46.63$ & $-68: 48: 47.1$ & 2,5 \\
ST12 & IRAS 05328-6827 & 5126566 & 2008 May 12 & $05: 32: 38.59$ & $-68: 25: 22.2$ & 3,6 \\
\hline
\end{tabular}

Notes. ${ }^{(a)}$ The source is in a cluster; ${ }^{(b)}$ 2MASS ID.

References. (1) Shimonishi et al. (2008); (2) Seale et al. (2009); (3) Oliveira et al. (2009); (4) Whitney et al. (2008); (5) Gruendl \& Chu (2009); (6) van Loon et al. (2010a).

Table 2. Details of detected ice features.

\begin{tabular}{ccccc}
\hline \hline Molecule & $\begin{array}{c}\text { Wavelength } \\
{[\mu \mathrm{m}]}\end{array}$ & Vibration Mode & $\begin{array}{c}A^{a} \\
{\left[10^{-17} \mathrm{~cm} \mathrm{molecule}{ }^{-1}\right]}\end{array}$ & Reference \\
\hline $\mathrm{H}_{2} \mathrm{O}$ & 3.05 & O-H stretch & 20 & 1 \\
$\mathrm{CH}_{3} \mathrm{OH}$ & 3.53 & C-H stretch & 0.76 & 2 \\
$\mathrm{CO}_{2}$ & 4.27 & C-O stretch & 7.6 & 1 \\
${ }^{13} \mathrm{CO}_{2}$ & 4.38 & ${ }^{13} \mathrm{C}-\mathrm{O}$ stretch & 7.8 & 1 \\
$\mathrm{XCN}$ & 4.62 & CN stretch & $\ldots$ & 3 \\
$\mathrm{CO}$ & 4.67 & C-O stretch & 1.1 & 1 \\
\hline
\end{tabular}

Notes. ${ }^{(a)}$ Band strengths of absorption features.

References. (1) Gerakines et al. (1995); (2) D’Hendecourt \& Allamandola (1986); (3) Schutte \& Greenberg (1997).

\section{Results}

\subsection{Observed spectra}

Observed spectra for all the objects are shown in Fig. 1 and details of the detected ice features are summarized in Tables 2 and 3. The absorption feature of the $3.05 \mu \mathrm{m} \mathrm{H}_{2} \mathrm{O}$ ice is detected toward all the objects except ST6 and ST9. The profile of the $\mathrm{H}_{2} \mathrm{O}$ ice feature is resolved, and this permits more accurate determination of the column density than in Shimonishi et al. (2008). One object (ST8) shows a structured profile of the $\mathrm{H}_{2} \mathrm{O}$ ice, which is often linked to the thermal processing of the ice mantles (Öberg et al. 2007). The water-ice band of ST6 is seen in the spectrum of Shimonishi et al. (2008), but it is not detected in the present spectra owing to the slightly poor sensitivity of the present observation mode. The $3 \mu \mathrm{m}$ region of ST9 overlaps prominent emission components, which makes it difficult to identify the underlying absorption bands around $3 \mu \mathrm{m}$. A broad depression is seen in the $4.3-4.7 \mu \mathrm{m}$ region of ST7. It can probably be attributed to a combination mode of the $\mathrm{H}_{2} \mathrm{O}$ ice, which typically peaks around $4.5 \mu \mathrm{m}$ (Gibb et al. 2000).

The absorption feature of the $4.27 \mu \mathrm{m} \mathrm{CO}_{2}$ ice is detected toward all the objects. Some objects show the absorption features of $3.53 \mu \mathrm{m} \mathrm{CH}_{3} \mathrm{OH}$ ice, $4.38 \mu \mathrm{m}{ }^{13} \mathrm{CO}_{2}$ ice, $4.62 \mu \mathrm{m} \mathrm{XCN}$, and the $4.67 \mu \mathrm{m} \mathrm{CO}$ ice. Since the $\mathrm{CO}_{2}$ and $\mathrm{CO}$ ice features are not completely resolved in our spectra, the widths of the observed features are larger than those of their typical laboratory spectra. The detections of the ${ }^{13} \mathrm{CO}_{2}$ ice and XCN features are still tentative because of the unresolved absorption bands of gaseous $\mathrm{CO}$, which sometimes appear in the wavelength region of these
Table 3. Detection of ice features in our YSO spectra.

\begin{tabular}{ccccccc}
\hline \hline Number & $\mathrm{H}_{2} \mathrm{O}$ & $\mathrm{CO}_{2}$ & $\mathrm{CO}$ & $\mathrm{CH}_{3} \mathrm{OH}$ & ${ }^{13} \mathrm{CO}_{2}$ & XCN \\
\hline ST1 & $\sqrt{ }$ & $\sqrt{ }$ & $\sqrt{ }$ & - & - & - \\
ST2 & $\sqrt{ }$ & $\sqrt{ }$ & - & $\sqrt{ }$ & $?$ & - \\
ST3 & $\sqrt{ }$ & $\sqrt{ }$ & $\sqrt{ }$ & - & - & - \\
ST4 & $\sqrt{ }$ & $\sqrt{ }$ & $\sqrt{ }$ & - & - & - \\
ST5 & $\sqrt{ }$ & $\sqrt{ }$ & $\sqrt{ }$ & $\sqrt{ }$ & - & - \\
ST6 & - & $\sqrt{ }$ & $\sqrt{ }$ & - & - & $?$ \\
ST7 & $\sqrt{ }$ & $\sqrt{ }$ & $\sqrt{ }$ & - & $\sqrt{ }$ & $?$ \\
ST8 & $\sqrt{ }$ & $\sqrt{ }$ & $\sqrt{ }$ & - & $\sqrt{ }$ & - \\
ST9 & $?$ & $\sqrt{ }$ & $\sqrt{ }$ & - & $?$ & - \\
ST10 & $\sqrt{ }$ & $\sqrt{ }$ & $\sqrt{ }$ & - & - & $?$ \\
ST11 & $\sqrt{ }$ & $\sqrt{ }$ & - & $?$ & - & - \\
ST12 & $\sqrt{ }$ & $\sqrt{ }$ & - & $?$ & - & - \\
\hline
\end{tabular}

Notes. $\sqrt{ }=$ clear detection, $?=$ tentative detection, $-=$ no detection.

features (Chiar et al. 1998; Boogert et al. 2000). The nondetection of the ${ }^{13} \mathrm{CO}_{2}$ ice in ST10, on the other hand, gives a rough lower limit of ${ }^{13} \mathrm{CO}_{2} /{ }^{12} \mathrm{CO}_{2}$ of about 95 . This is in the same range as the isotopic ratio for Galactic YSOs (Boogert et al. 2000). These minor features are labeled in Fig. 2 and summarized in Table 3.

Several emission features that were not resolved in the lowresolution spectra of Shimonishi et al. (2008) are detected in the present spectra. They are hydrogen recombination lines (2.62 $\mu \mathrm{m} \operatorname{Br} \beta, 3.05 \mu \mathrm{m} \operatorname{Pf} \epsilon, 3.29 \mu \mathrm{m} \operatorname{Pf} \delta, 3.74 \mu \mathrm{m} \mathrm{Pf} \gamma$, $4.05 \mu \mathrm{m} \operatorname{Br} \alpha, 4.64 \mu \mathrm{m} \operatorname{Pf} \beta)$ and polycyclic aromatic hydrocarbon (PAH) emission bands $(3.3 \mu \mathrm{m}$ and $3.4 \mu \mathrm{m})$. Detection of 

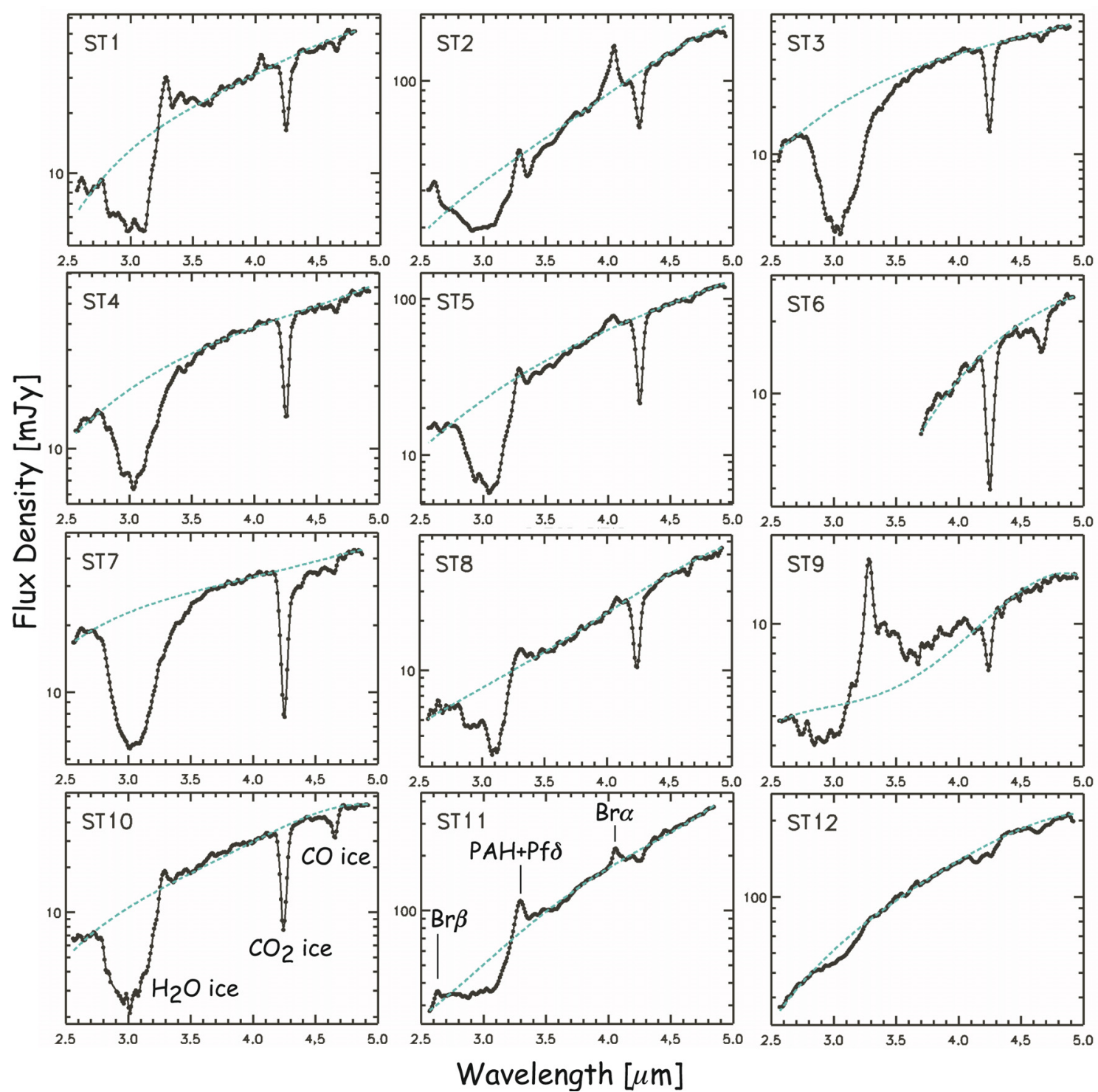

Fig. 1. AKARI IRC NG 2.5-5 $\mu \mathrm{m}$ spectra of YSOs in the LMC. Dashed lines represent derived continuum. The positions of detected features are labeled.

these emission features allows detailed discussions about the correlation between ice abundances and YSO characteristics (see Sect. 5.2).

\subsection{Spectral fitting and calculation of ice column densities}

To subtract the continuum, we fitted a polynomial of the second to fourth order to the continuum regions and derived the optical depth spectra. The wavelength regions used for the continuum were set to avoid a region with emission or absorption features, and they were typically $2.5-2.7 \mu \mathrm{m}, 4.1-4.15 \mu \mathrm{m}$, and 4.85-4.95 $\mu \mathrm{m}$. The absorption features of ices except $\mathrm{H}_{2} \mathrm{O}$ were not completely resolved with our spectral resolution. Therefore, to calculate ice column densities, we used different methods for the $\mathrm{H}_{2} \mathrm{O}$ ice and the other ices.

\subsection{1. $\mathrm{H}_{2} \mathrm{O}$ ice}

The spectral resolution of the present spectra is sufficient to resolve a broad feature of the $\mathrm{H}_{2} \mathrm{O}$ ice, and the true optical depth can be measured. Thus a direct comparison of laboratory spectra is possible. We fitted laboratory ice profiles to the observed spectra by a $\chi^{2}$ minimization method and derived the column density from the equation

$$
N=\int \tau \mathrm{d} v / A
$$

where $A$ is the band strength of each ice feature (in units of $\mathrm{cm}$ molecule $\mathrm{e}^{-1}$ ) as measured in the laboratory, $N$ the column density in $\mathrm{cm}^{-2}, v$ the wavenumber in units of $\mathrm{cm}^{-1}$, and $\tau$ the optical depth. The integration is performed over the $\mathrm{H}_{2} \mathrm{O}$ ice absorption band. The wavelength range used for the fit is set to avoid the $3.3 \mu \mathrm{m}$ PAH emission band and the bottom of the $\mathrm{H}_{2} \mathrm{O}$ absorption feature, which typically suffers from the weak background emission. Typically, 2.7-2.9 $\mu \mathrm{m}$ and $3.1-3.2 \mu \mathrm{m}$ are used for the fitting. A single laboratory ice profile of the pure $\mathrm{H}_{2} \mathrm{O}$ ice at $10 \mathrm{~K}$, which is expected to exist in the cold envelope of YSOs, is fitted to the observed spectra. The laboratory spectra of the $\mathrm{H}_{2} \mathrm{O}$ ice was taken from the 

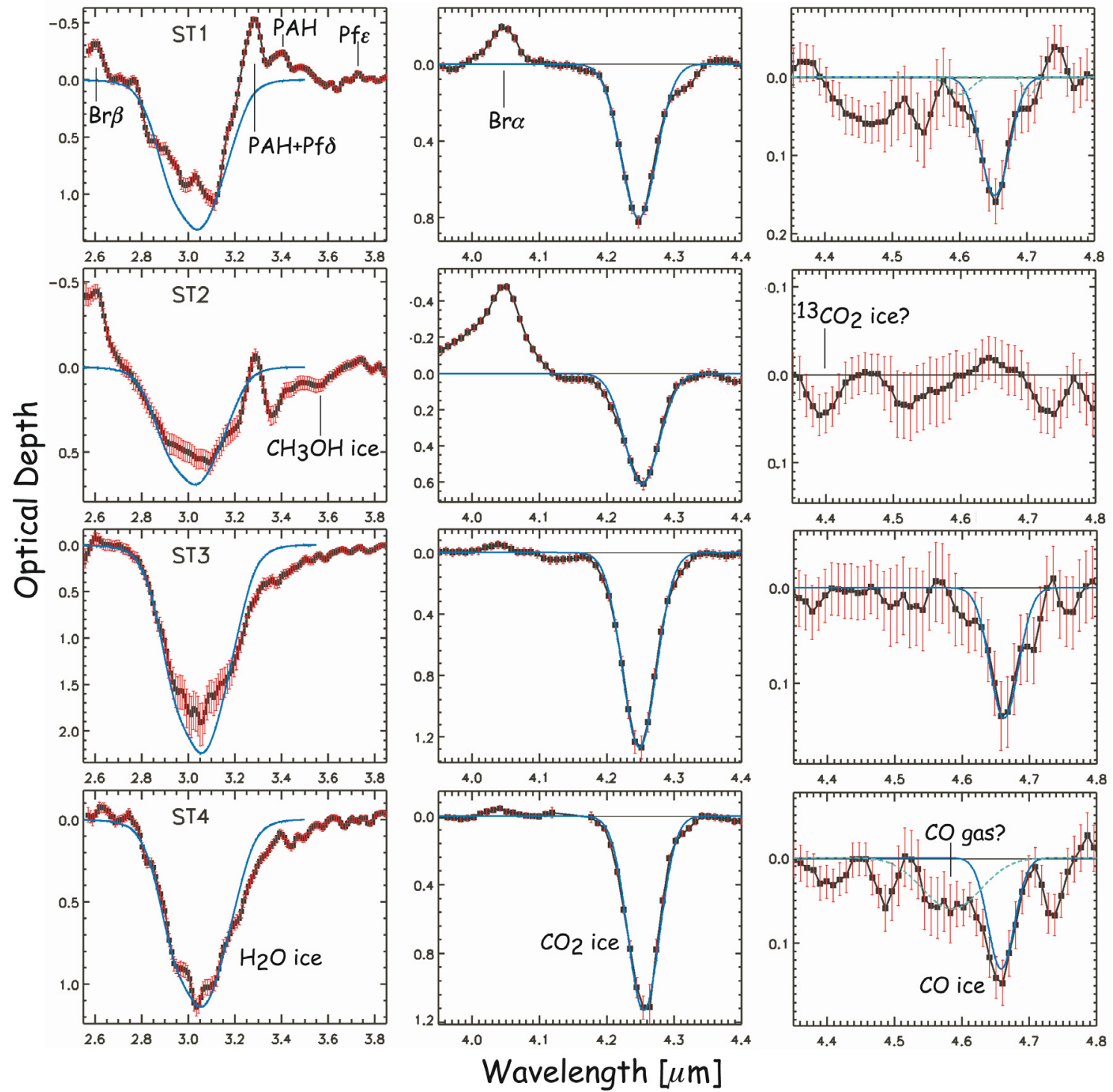

Fig. 2. Observed spectra of sample YSOs after the continuumd subtraction. A vertical axis represents the optical depth. The positions of the detected features are labeled. (Left) 2.6-3.8 $\mu$ m region. A solid line represents the fitted laboratory spectrum of the $\mathrm{H}_{2} \mathrm{O}$ ice $\left(\right.$ pure $\mathrm{H}_{2} \mathrm{O}$ at $10 \mathrm{~K}$, Ehrenfreund et al. 1996). 2.9-3.1 $\mu \mathrm{m}$ (bottom of the $\mathrm{H}_{2} \mathrm{O}$ ice feature) and 3.2-3.4 $\mu \mathrm{m}$ are removed from the fitting wavelength. (Center) 3.9-4.4 $\mu \mathrm{m}$ region. A solid line represents a fitted Gaussian profile for a measurement of the equivalent width of the $\mathrm{CO}_{2}$ ice feature. (Right) $4.4-4.8 \mu \mathrm{m}$ region. A dashed line indicates unresolved $\mathrm{CO}$ gas bands and the possible $\mathrm{XCN}$ feature, which were subtracted when fitting the $\mathrm{CO}$ ice feature (solid line).

Leiden molecular astrophysics database ${ }^{2}$ (Ehrenfreund et al. 1996). We adopted the band strengths of the $\mathrm{H}_{2} \mathrm{O}$ ice band as $2.0 \times 10^{-16} \mathrm{~cm} \mathrm{molecule}^{-1}$ (Gerakines et al. 1995). The derived column densities are listed in Table 4, and results of the fitting are shown in Fig. 2.

\subsection{2. $\mathrm{CO}_{2}$ ice and $\mathrm{CO}$ ice}

In case the spectral resolution is insufficient to resolve the absorption features, we cannot derive the "true" optical depth from the spectra and we can not use Eq. (1) to calculate column densities. Instead, we use the equivalent width of the absorption features to calculate column densities since it does not depend on the spectral resolution. The equivalent width $(E W)$ is defined as

$E W=\int F_{\text {norm }} \mathrm{d} \lambda$,

${ }^{2}$ Data available at http://www.strw. leidenuniv.nl/lab where $F_{\text {norm }}=1-F_{\text {obs }} / F_{\mathrm{c}}$,

and $F_{\text {norm }}$ is the normalized flux, $F_{\text {obs }}$ and $F_{\mathrm{c}}$ are the observed and continuum flux, and $\lambda$ is the wavelength. A Gaussian profile with the fixed central wavelength was fitted to the $\mathrm{CO}_{2}$ and $\mathrm{CO}$ absorption features to measure the equivalent width. To convert the equivalent width to the column density, we need to calculate the "curve-of-growth" of each absorption feature. The detailed discussion about our method is described in Appendix A. To calculate the curve-of-growth, we need to assume an intrinsic profile of the target absorption band. It is known that the absorption profiles of the $\mathrm{CO}_{2}$ and $\mathrm{CO}$ ices change depending on the temperature and compositions of the ices (Gerakines et al. 1999; Chiar et al. 1998). By considering the observed variation in ice profiles around massive YSOs, we choose appropriate profiles for the $\mathrm{CO}_{2}$ and $\mathrm{CO}$ ice and estimated the error of our analysis (see Appendix A for details). We adopted the band strengths of the $\mathrm{CO}_{2}$ and $\mathrm{CO}$ ices to be $7.6 \times 10^{-17}$ and $1.1 \times$ $10^{-17} \mathrm{~cm}$ molecule ${ }^{-1}$, respectively (Gerakines et al. 1995). 

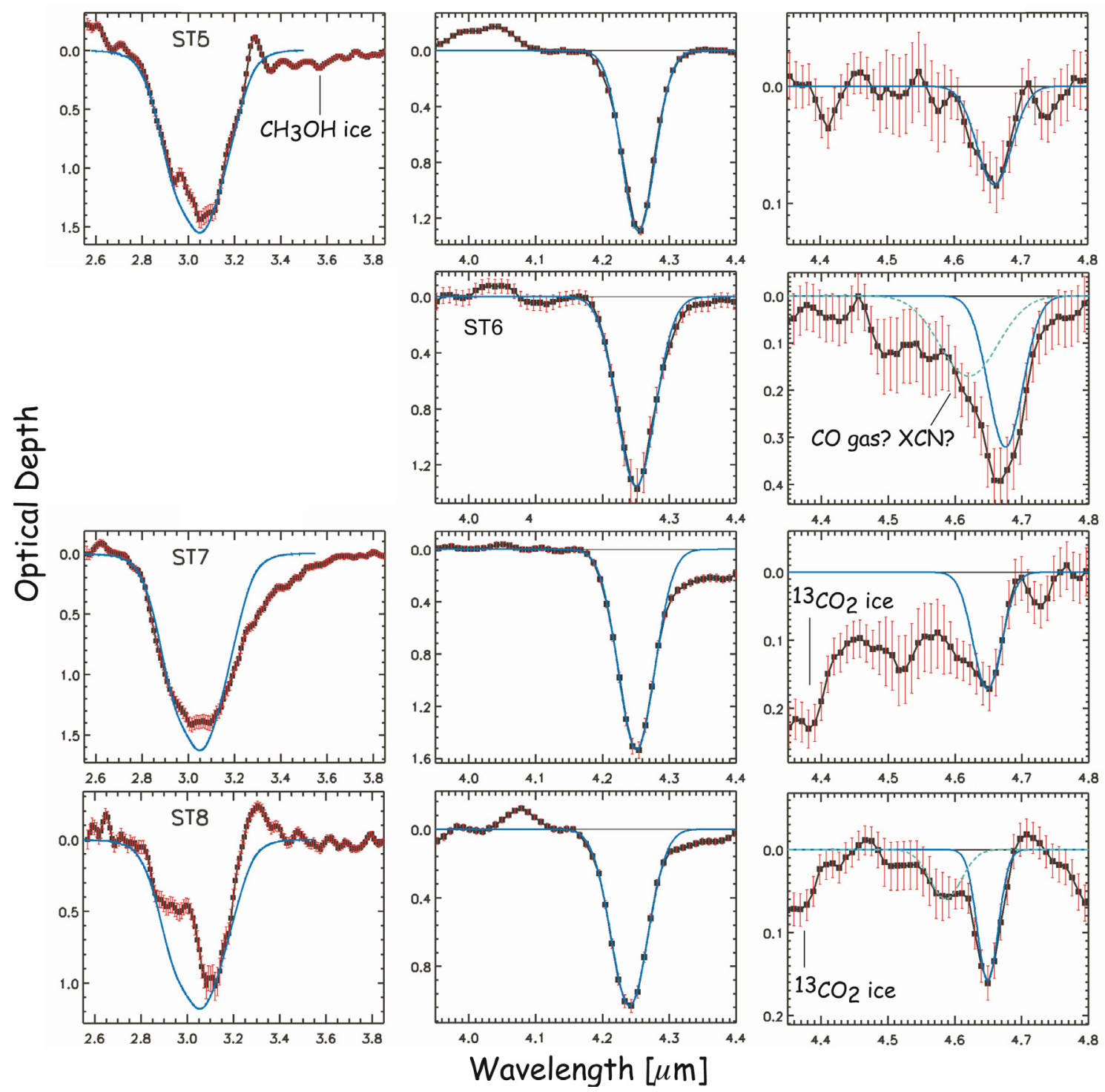

Fig. 2. continued.

Gas-phase $\mathrm{CO}_{2}$ shows narrow absorption lines around the wavelength range of the $\mathrm{CO}_{2}$ ice feature, and it is difficult to resolve it with our spectral resolution. However, the contribution of the gas-phase $\mathrm{CO}_{2}$ is generally small toward YSOs (Gerakines et al. 1999; Nummelin et al. 2001), and we assume that it has a negligible effect on the derived equivalent widths, except for ST12, which shows a strong absorption band of the gasphase $\mathrm{CO}_{2}$. On the other hand, the gas-phase $\mathrm{CO}$ ro-vibrational lines sometimes appear in the spectra of YSOs and are blended with the $\mathrm{CO}$ ice feature with our spectral resolution (Chiar et al. 1998). Unresolved gas-phase CO absorption bands are expected to cause shallow and broad absorption features on either or both sides of the $\mathrm{CO}$ ice feature. Therefore, in cases where these bands are seen on the sides of the $\mathrm{CO}$ ice feature, we subtracted them by assuming a Gaussian profile. The derived column densities are listed in Table 4.

\subsection{3. $\mathrm{CH}_{3} \mathrm{OH}$ ice, ${ }^{13} \mathrm{CO}_{2}$ ice, and $\mathrm{XCN}$}

In this study, we did not estimate the column densities of these minor ice species because the observed strengths of these ice features are too weak to discuss the column densities with the present $\mathrm{S} / \mathrm{N}$. In addition, it is difficult to distinguish the ${ }^{13} \mathrm{CO}_{2}$ ice and $\mathrm{XCN}$ features from the unresolved $\mathrm{CO}$ gas absorption bands by our spectral resolution. Observations of these minor ice features with higher spectral resolution and better $\mathrm{S} / \mathrm{N}$ are challenges for the future.

\section{Discussion}

\subsection{Large $\mathrm{CO}_{2} / \mathrm{H}_{2} \mathrm{O}$ ice ratio in the $L M C$}

The derived column densities of the $\mathrm{H}_{2} \mathrm{O}$ and $\mathrm{CO}_{2}$ ices are plotted in Fig. 3. The average $\mathrm{CO}_{2} / \mathrm{H}_{2} \mathrm{O}$ ice column density ratio of our samples is calculated to be $0.36 \pm 0.09$. The present samples are massive YSOs and should be compared to Galactic massive samples. Thus the column densities of Galactic massive YSOs $\left(\mathrm{CO}_{2} / \mathrm{H}_{2} \mathrm{O} \sim 0.17 \pm 0.03\right.$, Gerakines et al. 1999; Gibb et al. 2004) are also plotted in the figure for comparison. It is reported that Galactic quiescent molecular clouds show a similar $\mathrm{CO}_{2}$ ice abundance ( 0.18 \pm 0.04 , Whittet et al. 2007). As can be seen 
T. Shimonishi et al.: Ices around Embedded YSOs in the LMC
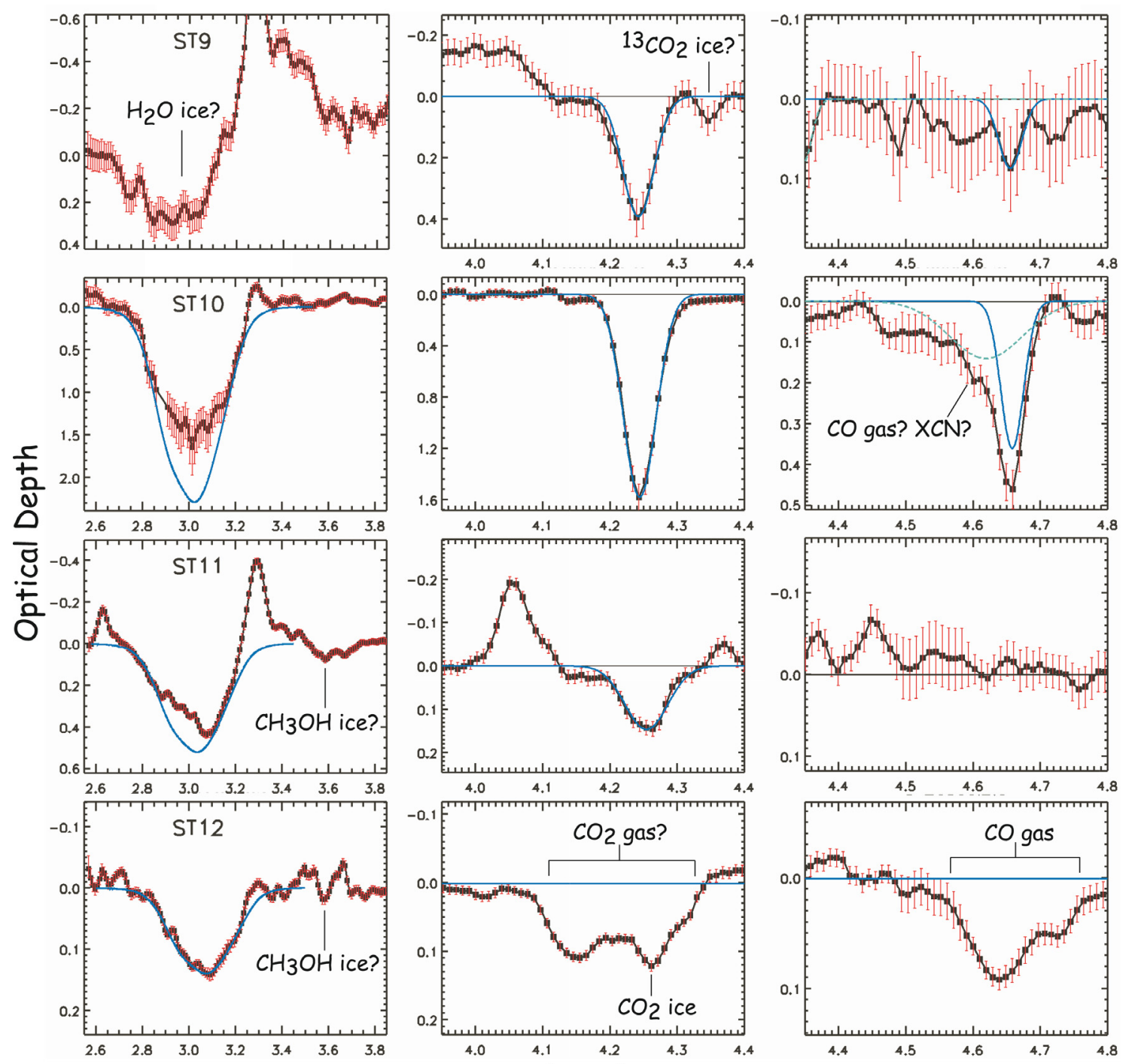

Wavelength $[\mu \mathrm{m}]$

Fig. 2. continued.

Table 4. Column density of ices and properties of YSOs.

\begin{tabular}{lccccccc}
\hline \hline Number & $\begin{array}{c}N\left(\mathrm{H}_{2} \mathrm{O}\right) \\
{\left[10^{17} \mathrm{~cm}^{-2}\right]}\end{array}$ & $\begin{array}{c}N\left(\mathrm{CO}_{2}\right) \\
{\left[10^{17} \mathrm{~cm}^{-2}\right]}\end{array}$ & $\begin{array}{c}N\left(\mathrm{CO}_{2}\right) / N\left(\mathrm{H}_{2} \mathrm{O}\right) \\
{[\%]}\end{array}$ & $\begin{array}{c}N(\mathrm{CO}) \\
{\left[10^{17} \mathrm{~cm}^{-2}\right]}\end{array}$ & $\begin{array}{c}N(\mathrm{CO}) / N\left(\mathrm{H}_{2} \mathrm{O}\right) \\
{[\%]}\end{array}$ & $\begin{array}{c}E W(\mathrm{Br} \alpha) \\
{[\AA]}\end{array}$ & $\begin{array}{c}L_{\text {tot }} \\
{\left[10^{3} \times L_{\odot}\right]}\end{array}$ \\
\hline ST1 & $22.5 \pm 0.96$ & $5.66 \pm 0.64$ & 25 & $3.62 \pm 0.93$ & 16 & $100 \pm 7$ & $53 \pm 18$ \\
ST2 & $11.9 \pm 1.0$ & $3.64 \pm 0.38$ & 31 & $<0.5$ & $<1$ & $343 \pm 12$ & $182 \pm 9$ \\
ST3 & $38.1 \pm 3.9$ & $10.6 \pm 1.6$ & 28 & $3.17 \pm 0.92$ & 8 & $21.3 \pm 6.3$ & $9.9 \pm 1.0$ \\
ST4 & $19.6 \pm 0.83$ & $7.41 \pm 0.79$ & 38 & $2.71 \pm 1.2$ & 14 & $17.4 \pm 5.1$ & $4.0 \pm 0.4$ \\
ST5 & $26.4 \pm 1.2$ & $10.1 \pm 0.79$ & 39 & $2.33 \pm 0.82$ & 9 & $119 \pm 7$ & $66 \pm 12$ \\
ST6 & $45.6 \pm 16^{a}$ & $17.1 \pm 3.0$ & 37 & $<13$ & $\ldots$ & $44.3 \pm 21$ & $6.6 \pm 2.5$ \\
ST7 & $27.8 \pm 0.95$ & $19.5 \pm 1.8$ & 70 & $<4$ & $<14$ & $15.8 \pm 6.1$ & $20 \pm 5$ \\
ST8 & $20.1 \pm 1.2$ & $9.34 \pm 1.1$ & 47 & $2.60 \pm 0.75$ & 13 & $64.1 \pm 6.2^{c}$ & $46 \pm 16^{d}$ \\
ST9 & $\ldots$ & $1.84 \pm 0.17$ & $\ldots$ & $<2$ & $\ldots$ & $\ldots$ & $54 \pm 22$ \\
ST10 & $39.8 \pm 5.0$ & $14.5 \pm 2.1$ & 36 & $7.72 \pm 1.5$ & 19 & $<10$ & $32 \pm 14$ \\
ST11 & $8.9 \pm 0.3$ & $0.9 \pm 0.23$ & 10 & $<0.5$ & $<1$ & $116 \pm 6$. & $338 \pm 27$ \\
ST12 & $2.34 \pm 0.12$ & $0.9 \pm 0.15^{b}$ & 39 & $\ldots$ & $\ldots$ & $<7$ & $23 \pm 3^{d}$ \\
\hline
\end{tabular}

Notes. ${ }^{(a)}$ Derived from low-resolution spectrum of Shimonishi et al. (2008), but re-calculated after applying our own spectral reduction pipeline.

(b) Derived by Oliveira et al. (2009).

${ }^{(c)}$ Detection of a strong [OIII] emission line at $88 \mu \mathrm{m}$ is reported by van Loon et al. (2010a).

(d) Estimated luminosities 95 and $62 L_{\odot}$ from van Loon et al. (2010a), on the basis of far-infrared data, for ST8 and ST10, respectively. 


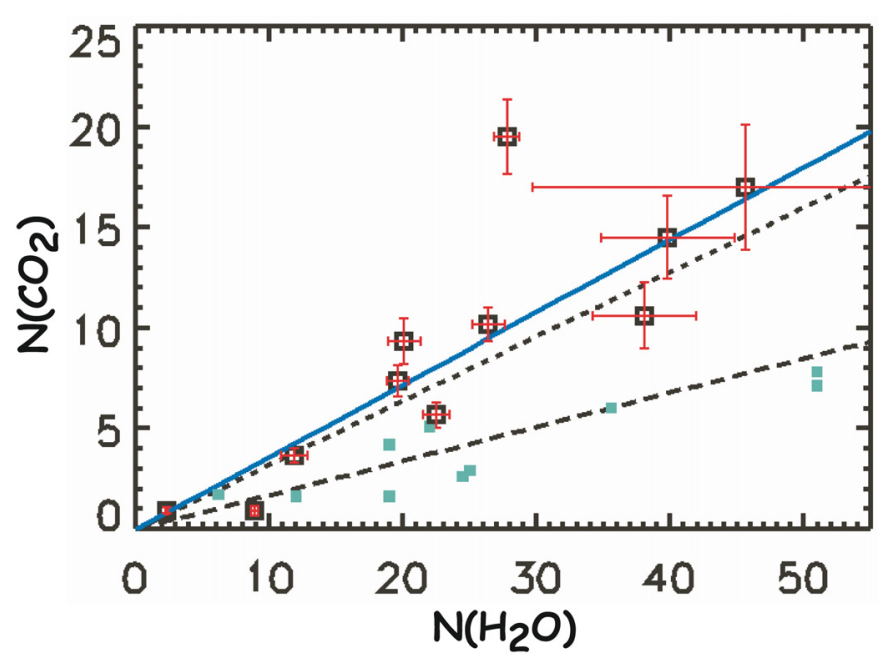

Fig. 3. $\mathrm{CO}_{2}$ ice vs. $\mathrm{H}_{2} \mathrm{O}$ ice column density in units of $10^{17} \mathrm{~cm}^{-2}$. Open squares with error bars represent the results of this study. Filled squares represent those of Galactic massive YSOs (Gerakines et al. 1999; Gibb et al. 2004). An average $\mathrm{CO}_{2} / \mathrm{H}_{2} \mathrm{O}$ ice ratio of our samples $(0.36)$ is plotted as a solid line. Dotted and dashed lines represent $\mathrm{CO}_{2} / \mathrm{H}_{2} \mathrm{O}$ ratio $\sim 0.32$ and 0.17 , which correspond to the typical $\mathrm{CO}_{2} / \mathrm{H}_{2} \mathrm{O}$ ratio of Galactic low- and intermediate-mass YSOs, and massive YSOs, respectively. The dotted line also corresponds to the $\mathrm{CO}_{2} / \mathrm{H}_{2} \mathrm{O}$ ratio of the LMC's massive YSOs derived by Oliveira et al. (2009).

in the figure, the typical abundance ${ }^{3}$ of the $\mathrm{CO}_{2}$ ice toward the LMC's massive YSOs is larger than for Galactic massive YSOs. Also, the scatter of the $\mathrm{CO}_{2} / \mathrm{H}_{2} \mathrm{O}$ ratio is larger than for Galactic samples.

Shimonishi et al. (2008) reported that the typical $\mathrm{CO}_{2} / \mathrm{H}_{2} \mathrm{O}$ ice ratio in the LMC is $0.45 \pm 0.17$ based on the low-resolution spectra of the LMC's YSO (targets included in the present samples). The present result is consistent with the $\mathrm{CO}_{2} / \mathrm{H}_{2} \mathrm{O}$ ratio of Shimonishi et al. (2008) within the uncertainties, but it is slightly lower than our previous result. It is mostly attributed to the effect of the PAH $3.3 \mu \mathrm{m}$ emission, which was not resolved and thus not correctly counted in the previous study. Oliveira et al. (2009) have estimated the column densities of the $\mathrm{CO}_{2}$ ice for some common objects with the present targets based on the $15.2 \mu \mathrm{m}$ feature and also revised some of the column density estimates of Shimonishi et al. (2008). The $\mathrm{CO}_{2}$ ice column densities estimated in Oliveira et al. (2009) are slightly lower than our estimate in Table 4, but generally consistent within the uncertainties. They also suggest that the $\mathrm{CO}_{2}$ ice abundance of the LMC samples is higher than in massive YSOs in our Galaxy $\left(\mathrm{CO}_{2} / \mathrm{H}_{2} \mathrm{O} \sim 0.32\right.$, Fig. 3).

The high $\mathrm{CO}_{2} / \mathrm{H}_{2} \mathrm{O}$ ratio in the LMC indicated by the previous studies is now confirmed with the increased number of samples. In addition, the uncertainties of the derived column densities decreased from those of Shimonishi et al. (2008) thanks to the higher $\mathrm{S} / \mathrm{N}$, the higher spectral resolution, and the improved treatment of the curve-of-growth method. It is also worth mentioning that a relatively high $\mathrm{CO}_{2} / \mathrm{H}_{2} \mathrm{O}$ ratio of $0.32 \pm 0.02$ is observed toward Galactic low- and intermediate-mass YSOs (Pontoppidan et al. 2008). Their samples also show a large scatter in the $\mathrm{CO}_{2}$ ice abundance as seen in the present samples. A similar trend in the $\mathrm{CO}_{2}$ ice abundance between the LMC's massive YSOs and Galactic low- and intermediate-mass

\footnotetext{
3 The "abundance" of a given ice is generally defined as the ratio of the column density relative to the $\mathrm{H}_{2} \mathrm{O}$ ice since it is the most abundant ice species.
}

YSOs may indicate some similarities of star formation conditions between these different samples, but a qualitative explanation is an issue for future investigation.

The derived $\mathrm{CO} / \mathrm{H}_{2} \mathrm{O}$ ice ratio of the LMC's YSO ranges from $\sim 0.01$ to 0.2 (Table 4), and this is similar to what is observed toward Galactic massive YSOs $(\sim 0.02-0.2$, Chiar et al. 1998; Gibb et al. 2004). Also, the distribution range of the $\mathrm{H}_{2} \mathrm{O}$ ice column densities of our samples is nearly comparable to that of the Galactic YSOs. Therefore, we can assume that there should be a different physical or chemical environment that selectively enhances the production of $\mathrm{CO}_{2}$ ice in the $\mathrm{LMC}$.

It is widely accepted that the $\mathrm{CO}_{2}$ ice in the cold envelope of a YSO is formed by grain surface reactions since gas-phase reactions are not able to produce the observed abundance of the $\mathrm{CO}_{2}$ ice, while molecules like $\mathrm{CO}$ are mainly formed by the gasphase reaction (e.g., Millar et al. 1991). However, the formation mechanism of the $\mathrm{CO}_{2}$ ice still remains unclear, and it is one of the key topics in astrochemistry. Laboratory experiments indicate that the $\mathrm{CO}_{2}$ ice is efficiently produced by the UV photon irradiation to $\mathrm{H}_{2} \mathrm{O}-\mathrm{CO}$ ice mixtures (e.g., Gerakines et al. 1996; Watanabe et al. 2007). It is reported by several authors that the LMC has an order-of-magnitude stronger UV radiation field than our Galaxy owing to its active star formation and its metal-poor environment (Israel et al. 1986; Lee et al. 2007). Thus the strong UV radiation field is able to help the efficient formation of the $\mathrm{CO}_{2}$ ice in the LMC.

On the other hand, an alternative mechanism of the $\mathrm{CO}_{2}$ ice formation has been proposed to explain the detection of the abundant $\mathrm{CO}_{2}$ ice toward quiescent molecular clouds, where energy sources of UV photon are not expected (Whittet et al. 1998). A theoretical study suggests that the $\mathrm{CO}_{2}$ ice can also be formed through the diffusive surface chemistry without UV irradiation (Ruffle \& Herbst 2001). This model suggests that a sufficient abundance of the $\mathrm{CO}_{2}$ ice can be produced at relatively high dust temperatures, but this process is quite sensitive to the dust temperature and other assumed conditions. Several studies report that the interstellar dust temperature in the LMC is generally higher than in our Galaxy based on far-infrared to submillimeter observations of diffuse emission (e.g., Aguirre et al. 2003; Sakon et al. 2006). In addition, van Loon et al. (2010a,b) derived the dust temperatures of YSOs in the LMC and Small Magellanic Cloud (SMC) and suggest that the dust temperatures become higher in more metal-poor environments of the SMC. Thus the dust temperatures of the present samples may be systematically higher than their Galactic counterparts, which are in more metal-rich environments. Therefore the high dust temperature in the LMC can also be a possible cause for the enhanced production of the $\mathrm{CO}_{2}$ ice. From the discussion, we conclude that the general properties of the LMC's environments, the strong UV radiation field, and the high dust temperature are responsible for the observed high $\mathrm{CO}_{2}$ ice abundance.

One of the interstellar molecular features that likely develop from UV photolysis is the $4.62 \mu \mathrm{m}$ "XCN" feature, which is probably due to the $\mathrm{OCN}^{-}$(e.g., Pendleton et al. 1999; Bernstein et al. 2000). This feature provides a diagnostic of the local radiation field of objects (Spoon et al. 2003). In our galaxy, the $\mathrm{XCN}$ feature is generally very weak or absent toward YSOs and dark clouds, but is very strong toward a few high-mass YSOs and Galactic center objects (Gibb et al. 2004). Thus this feature could suggest that the LMC's strong UV radiation field is a dominant factor for the high $\mathrm{CO}_{2}$ ice abundance in the LMC. Some of our samples show a hint of this XCN feature on the shorter side of the CO ice feature (ST6, 7, 10). There may also be an additional component seen in the spectrum of ST4 and 

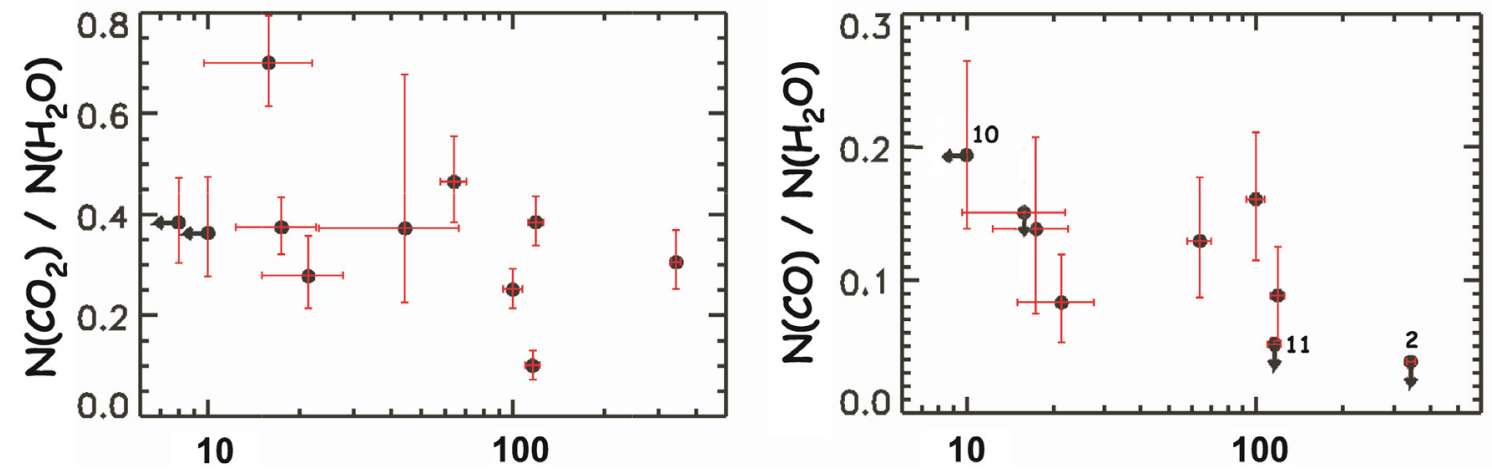

Equivalent Width $(\operatorname{Br} \alpha)[\AA]$

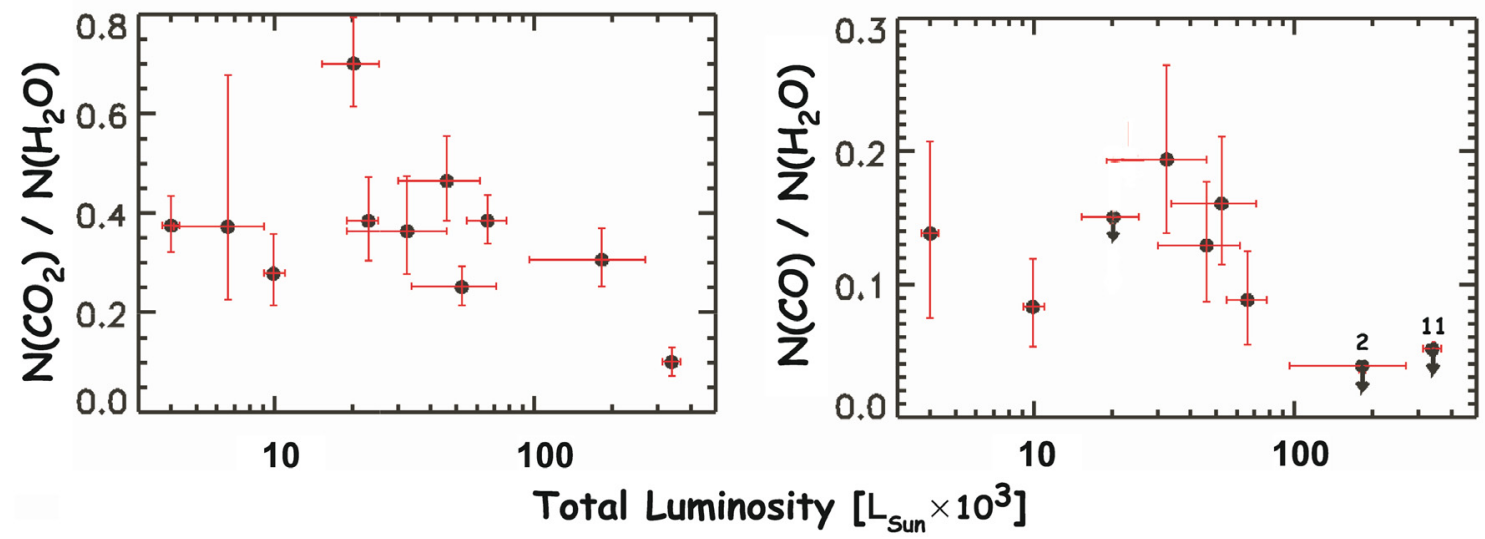

Fig. 4. The equivalent width of the hydrogen recombination line $(\mathrm{Br} \alpha)$ vs. $\mathrm{CO}_{2} / \mathrm{H}_{2} \mathrm{O}$ ratio (upper left) and $\mathrm{CO} / \mathrm{H}_{2} \mathrm{O}$ ratio (upper right). And a total luminosity of sample YSOs vs. $\mathrm{CO}_{2} / \mathrm{H}_{2} \mathrm{O}$ ratio (bottom left) and $\mathrm{CO} / \mathrm{H}_{2} \mathrm{O}$ ratio (bottom right). A YSO number given in Table 1 is plotted for the objects discussed in the text. A value of ST6 is not plotted due to its large uncertainties.

ST8, but the peak wavelength seems to be slightly shorter than the $\mathrm{XCN}$ feature. No clear trends are seen in the $\mathrm{CO}_{2} / \mathrm{H}_{2} \mathrm{O}$ ratio of the objects with possible detection of the XCN. However, we cannot separate this feature from unresolved $\mathrm{CO}$ gas lines because the spectral resolution is not high enough, and the detection of $\mathrm{XCN}$ is still tentative, so that no definite conclusion can be drawn from the present spectra. Future observations should provide the needed resolution.

\subsection{Correlation of ice abundance with YSO properties}

As described in Sect. 2, the present samples basically possess similar characteristics (high-mass Class I objects). Despite this, column densities of the $\mathrm{H}_{2} \mathrm{O}, \mathrm{CO}_{2}$ and $\mathrm{CO}$ ices and their ratio show large variations. To investigate which physical condition is dominant in the formation and evolution of ices, we compare the chemical conditions of ices and the properties of individual YSOs. It is probable that the radiation from the central star dominates the circumstellar environment of a massive YSO. If the formation of ices is controlled by the radiation from the star, there should be some parameters of YSOs that correlate well with the ice abundances.

First, we compared the strength of the hydrogen emission lines with the $\mathrm{CO}_{2}$ and $\mathrm{CO}$ ice abundance. Although it is not seen in the low-resolution spectra of Shimonishi et al. (2008), the present spectra clearly detect hydrogen recombination lines toward the target YSOs. It is known that massive YSOs in their more evolved stage ionize their circumstellar gas by stellar UV radiation and form compact HII regions around the central star (van den Ancker et al. 2000). A variation in their hydrogen line strength thus indicates that the present targets are in their various evolutionary stages and possess different radiation environments (especially as for UV) in their circumstellar region, as discussed in Seale et al. (2009). We measured the equivalent width of the $\operatorname{Br} \alpha$ line at $4.05 \mu \mathrm{m}$, which is the strongest hydrogen recombination line in the wavelength range of our observations.

Figure 4 shows the comparison of the equivalent width of the $\mathrm{Br} \alpha$ line with the $\mathrm{CO}_{2} / \mathrm{H}_{2} \mathrm{O}$ and $\mathrm{CO} / \mathrm{H}_{2} \mathrm{O}$ ice ratio. It seems that the abundance of the $\mathrm{CO}_{2}$ ice does not correlate with the strength of the $\mathrm{Br} \alpha$ line. The objects with similar $\mathrm{CO}_{2}$ abundance show various $\operatorname{Br} \alpha$ line strengths. This result indicates that the UV radiation from the central star is a less dominant factor for the evolution of the $\mathrm{CO}_{2}$ ice. However, in the previous section, we mentioned that the UV irradiation can help form $\mathrm{CO}_{2}$ ice. Several authors have demonstrated by simple calculation that the interstellar radiation field can penetrate the site where the $\mathrm{CO}_{2}$ ice forms (e.g., Whittet et al. 1998; Watanabe et al. 2007). We therefore suggest that the external (interstellar) UV radiation field may play an important role in $\mathrm{CO}_{2}$ ice formation. On the other hand, it seems that objects with strong $\operatorname{Br} \alpha$ line strengths (ST2, ST11) show a small CO ice abundance (upper limit), and one with the weakest $\mathrm{Br} \alpha$ line (ST10) shows the highest $\mathrm{CO}$ ice abundance. Although the $\mathrm{CO}$ ice abundance of relatively weak $\operatorname{Br} \alpha$ objects is rather scattered, the result indicates that the $\mathrm{CO}$ ice around YSOs is more likely to be affected by the stellar radiation than the $\mathrm{CO}_{2}$ ice.

A similar comparison was also made for the total luminosity of the YSOs, which is also a good estimate of the radiation environment of a YSO. The total luminosity was derived by the SED fitting described in Sect. 2. As seen in Fig. 4, no clear correlation 
is seen between the $\mathrm{CO}_{2}$ ice abundance and total luminosity of YSOs. The trend seen in the plot of the $\mathrm{CO}$ ice against the total luminosity is somewhat similar to what is seen in the comparison with the $\operatorname{Br} \alpha$ strength; i.e., the overall correlation is weak, but the most luminous objects in the present sample (ST2 and ST11) show a small abundance of CO.

The low $\mathrm{CO}$ ice abundance toward luminous and probably evolved (indicated by strong $\operatorname{Br} \alpha$ emission) YSOs can be explained by the low sublimation temperature of the $\mathrm{CO}$ ice. The sublimation temperature of the pure $\mathrm{CO}$ ice is lower than that of the pure $\mathrm{H}_{2} \mathrm{O}$ and $\mathrm{CO}_{2}$ ices $(16 \mathrm{~K}, 50 \mathrm{~K}$, and $90 \mathrm{~K}$, for $\mathrm{CO}$, $\mathrm{CO}_{2}$, and $\mathrm{H}_{2} \mathrm{O}$, Tielens 2005). Although the sublimation temperature changes depending on the chemical compositions of ices, their general trend does not change. The $\mathrm{CO}$ ice is therefore more easily affected by the stellar radiation owing to its low sublimation temperature. Given a similar geometry and distance between the central star and the circumstellar dust, the dust around more luminous YSOs is expected to be warmer since the radiation from the central star is the dominant heating source of their circumstellar dust. Likewise, dust around more evolved objects is expected to be warmer thanks to the dissipation of the circumstellar dust. The relatively high luminosity of ST2 and ST11 suggests that these objects might be clusters. However, we regard them here as YSOs of a similar kind as in other samples, because their infrared SEDs are well-fitted by the single YSO model of Robitaille et al. (2007), as well as in other samples. We thus suggest that the $\mathrm{CO}$ ice around the present luminous samples (ST2 and ST11) may be sublimated thanks to the intense radiation from the central star. The $\mathrm{CO}$ abundance of less luminous objects also has a scatter, which suggests that other factors than the stellar radiation may be important for the $\mathrm{CO}$ ice chemistry as for these objects.

\section{Summary}

To understand the chemical conditions of ices around YSOs in the Large Magellanic Cloud, we performed spectroscopic observations toward massive embedded YSOs and the candidates using the IRC onboard the infrared satellite AKARI. We obtained near-infrared spectra $(R \sim 80,2.5-5 \mu \mathrm{m})$ of 12 objects in total. The ice absorption features of $\mathrm{H}_{2} \mathrm{O}, \mathrm{CH}_{3} \mathrm{OH}, \mathrm{CO}_{2},{ }^{13} \mathrm{CO}_{2}$, $\mathrm{CO}$, and possibly XCN were detected in the spectra. In addition, hydrogen recombination lines and $\mathrm{PAH}$ emission bands were detected toward the majority of the targets.

Compared to our previous study (Shimonishi et al. 2008), the increased number of LMC's YSO samples, the higher spectral resolution, and the improved treatment of the curve-of-growth method make it possible to derive column densities of ices and their typical abundances more accurately. Also, the detection of hydrogen emission lines and the SED analysis of individual YSOs allow discussion of the correlation between ice abundances and YSO properties.

We derived the column densities of the $\mathrm{H}_{2} \mathrm{O}, \mathrm{CO}_{2}$ and $\mathrm{CO}$ ices by fitting the laboratory ice spectra (for $3.05 \mu \mathrm{m} \mathrm{H}_{2} \mathrm{O}$ ice feature) and by the curve-of-growth method (for $4.27 \mu \mathrm{m} \mathrm{CO}_{2}$ and $4.67 \mu \mathrm{m} \mathrm{CO}$ ice features). As a result, it is shown that the typical $\mathrm{CO}_{2} / \mathrm{H}_{2} \mathrm{O}$ ice ratio of our samples $(\sim 0.36 \pm 0.09)$ is higher than that of Galactic massive YSOs $(\sim 0.17)$, while the $\mathrm{CO} / \mathrm{H}_{2} \mathrm{O}$ ice ratio is comparable to Galactic ones. The systematical difference in the $\mathrm{CO}_{2}$ ice abundance around the LMC's massive YSOs, which was suggested by previous studies (Shimonishi et al. 2008; Oliveira et al. 2009), is now confirmed with the new near-infrared data. It is also shown that the scatter of the $\mathrm{CO}_{2} / \mathrm{H}_{2} \mathrm{O}$ ratio in the $\mathrm{LMC}$ is larger than for Galactic ones.
Although the formation mechanisms of the $\mathrm{CO}_{2}$ ice is still unclear, we suggest that the strong ultraviolet radiation field and/or the high dust temperature in the LMC are responsible for the observed high abundance of the $\mathrm{CO}_{2}$ ice. Our result provides evidence that YSOs in the metal-poor LMC maintain different chemical conditions in their circumstellar environments.

A relatively high $\mathrm{CO}_{2}$ ice abundance $(\sim 0.32)$ and a large scatter are also reported toward Galactic low- and intermediatemass YSOs. A similar trend of the $\mathrm{CO}_{2}$ ice abundance between the LMC's massive YSOs and Galactic low- and intermediatemass YSOs may indicate some commonality in these objects, but a qualitative explanation considering both the structure of a YSO envelope and the ice chemistry is required in the future.

The present YSO samples possess similar characteristics in their infrared SEDs, but they show a large variation in the $\mathrm{CO}_{2}$ and $\mathrm{CO}$ ice abundances and in their near-infrared emission features. We compared the $\mathrm{CO}_{2}$ and $\mathrm{CO}$ ice abundances with the strength of the hydrogen recombination line and total luminosity of the sample YSOs. It is shown that the $\mathrm{CO}_{2}$ ice abundance and these YSO characteristics do not show a correlation. The present result indicates that the internal stellar radiation does not play an important role in the evolution of the $\mathrm{CO}_{2}$ ice around a massive YSO. We thus need further investigations to shed light on the dominant physical factors in the $\mathrm{CO}_{2}$ ice formation. Similar to the $\mathrm{CO}_{2}$ ice, a clear correlation is not seen between the $\mathrm{CO}$ ice abundance and the YSO properties. However, it is suggested that the $\mathrm{CO}$ ice abundance of luminous and probably evolved samples are significantly lower than other samples. We infer that more volatile molecules like $\mathrm{CO}$ are susceptible to the effect of the stellar radiation.

The present results revealed the presence of a massive YSO that shows a $\mathrm{CO}_{2} / \mathrm{H}_{2} \mathrm{O}$ ratio of up to $\sim 0.7$. If there is also a chemical difference in the LMC's lower mass YSOs, it will indirectly tell us the chemical diversity of subsequently-formed planets since the interstellar ices are thought to be an origin of planetary system ices, as discussed in Ehrenfreund \& Schutte (2000). The future infrared telescope missions SPICA, JWST, and SOFIA will allow better understanding of the diversity of solid materials in the universe.

Acknowledgements. This work is based on observations with AKARI, a JAXA project with the participation of ESA. We are deeply grateful to the referee J. Th. van Loon for his useful comments, which greatly improved this paper. We thank all the members of the AKARI project for their continuous help and support. We are grateful to Youichi Ohyama for kind support in the analysis of spectroscopic data. We also thank Helen Fraser and Jennifer Noble for fruitful discussions in Glasgow. This research has made extensive use of the Leiden University's Laboratory ice database. This work is supported by a Grant-in-Aid for Scientific Research from the JSPS (No. 180204014).

\section{Appendix A: The curve-of-growth method}

We calculated column densities of unresolved ice features by using the equivalent width. This calculation technique is called the "curve-of-growth" method, and it is traditionally used to study gaseous absorption lines (Shore 2002). Since the optical depth is the logarithm conversion of the normalized flux $\left(1-F_{\mathrm{obs}} / F_{\mathrm{c}}\right)$, the peak optical depth derived from unresolved features is not a true value. Thus, in case of our low-resolution spectra, we will significantly underestimate a column density if it is derived from the simple integration of the optical depth as expressed 

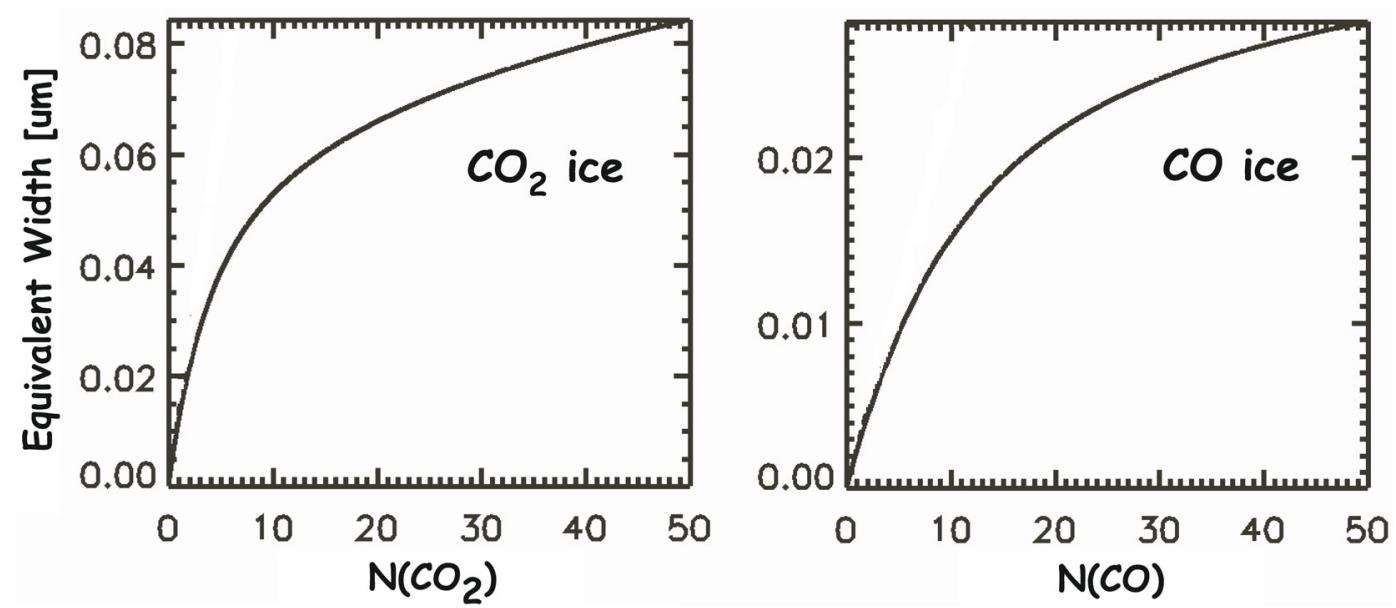

Fig. A.1. Calculated curve-of-growth for the $\mathrm{CO}_{2}$ ice (left) and the $\mathrm{CO}$ ice (right). These plots are used to convert measured equivalent widths to column densities. The uncertainties of derived column densities increased for deeper absorptions.

in Eq. (1), and this is why we need to use the curve-of-growth method. This method was applied for the first time to absorption features of ices in Shimonishi et al. (2008), but we overestimate the uncertainties and a detailed description of the method was not given because of space limitations. In this appendix, we describe our improved treatment of the curve-of-growth method.

First, we convert the measured equivalent width into a column density. To do this, we need to assume intrinsic absorption profiles of target ice features. Here, they are the $4.27 \mu \mathrm{m} \mathrm{CO}_{2}$ ice and $4.67 \mu \mathrm{m} \mathrm{CO}$ ice feature. For $\mathrm{CO}_{2}$, we considered the typical compositions and temperature of the interstellar $\mathrm{CO}_{2}$ ice and chose an appropriate laboratory spectrum as shown in Table A.1 (Ehrenfreund et al. 1996, data taken from the Leiden molecular astrophysics database). For $\mathrm{CO}$, it is reported that several combinations of laboratory ice profiles can account for the same observed spectrum and the fitting degenerates (Pontoppidan et al. 2003). We therefore used a single Gaussian profile that is close to the typically observed $\mathrm{CO}$ ice feature around massive YSOs (Chiar et al. 1998). The assumed profiles of the $\mathrm{CO}_{2}$ and $\mathrm{CO}$ ices features are summarized in Table A.1. By using these profiles, we calculated both equivalent widths and column densities for the various optical depths. The plot of the derived equivalent widths vs. column densities is shown in Fig. A.1, and this is called the curve-of-growth. The equivalent widths can be measured from observations, and these plots are used to derive column densities of ices; however, the uncertainty becomes larger for the higher column densities owing to the saturation effect of the curve-of-growth.

It is known that the actual profile of the observed ices in starforming regions is not uniform since they vary depending on the compositions and temperature of ices (Gerakines et al. 1999; Gibb et al. 2004). The derived column densities depend on the assumed profiles, so we need to check the validity of our method and to evaluate the uncertainties caused by the method.

We first prepared 2-5 $\mu \mathrm{m}$ ISO-SWS spectra of embedded massive YSOs $^{4}$ (Sloan et al. 2003), whose ice column densities are already presented in Gibb et al. (2004). The spectral resolution of these data is high enough to resolve narrow ice features $(R \sim 500-1000)$. Then their spectral resolution was degraded to the resolution of the AKARI IRC NG mode $(R \sim 80)$ by

\footnotetext{
${ }^{4}$ Data taken from http://isc.astro.cornell.edu/sloan/ library/swsatlas/atlas.html
}

Table A.1. Ice profiles used for calculating the curve-of-growth.

\begin{tabular}{cc}
\hline \hline Molecule & Ice profile \\
\hline $\mathrm{CO}_{2}$ & $\mathrm{H}_{2} \mathrm{O}: \mathrm{CO}_{2}$ ice mixture at $14 \mathrm{~K}$ \\
$\mathrm{CO}$ & Gaussian profile $\left(F W H M=6.89 \mathrm{~cm}^{-1}, \lambda_{\text {center }}=4.67 \mu \mathrm{m}\right)$ \\
\hline
\end{tabular}

convolving the point spread function of the IRC. Next, we derived column densities of the $\mathrm{CO}_{2}$ and $\mathrm{CO}$ ice by these converted spectra using the same method as that described in Sect. 4.2.2. The derived column densities and the results of Gibb et al. (2004) are plotted in Fig. A.2.

The figure indicates that the column densities derived from our curve-of-growth method are basically in good agreement with the results of Gibb et al. (2004), which derived the column densities from the high-resolution spectra. But a systematic difference and scatter are also seen in the figure. These are mainly caused by the difference between the observed and assumed ice profiles. The presence of weak emission or absorption features may also be responsible for these differences. We obtained the following equations from a straight-line fit to the data points in Fig. A.2.

$N\left(\mathrm{CO}_{2}\right)=N_{\mathrm{G} 04} \times(0.98 \pm 0.038)$

and

$N(\mathrm{CO})=N_{\mathrm{G} 04} \times(1.08 \pm 0.065)$

where $N_{\mathrm{G} 04}$ represents the column density of each ice presented in Gibb et al. (2004). In Shimonishi et al. (2008), we adopted $20 \%$ uncertainties to account for the above systematic difference in the derived column density of the $\mathrm{CO}_{2}$ ice and added to the observational uncertainties. The uncertainties were, however, overestimated as expressed in Eq. (A.1). In this study, the column densities derived from the curve-of-growth method are divided by the slope of the corresponding line to correct the systematic difference in the derived column densities. In addition, a $1-\sigma$ fitting error of the slope is considered as an uncertainty of our curve-of-growth method and added to the uncertainties in the observation and the fitting. 

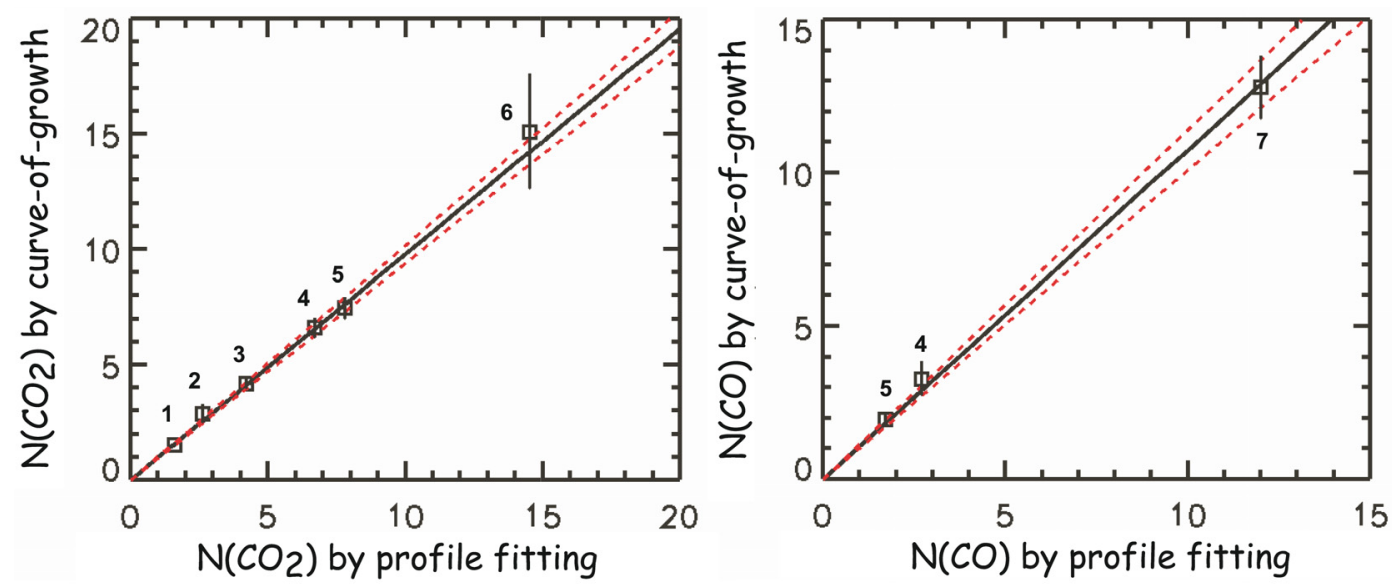

Fig. A.2. Comparison of the $\mathrm{CO}_{2}$ and $\mathrm{CO}$ ice column densities derived from the low-resolution spectra by the curve-of-growth and those derived from the high-resolution ISO-SWS spectra by the laboratory ice fitting (Gerakines et al. 1995; Gibb et al. 2004). The axes are expressed in units of $10^{17} \mathrm{~cm}^{-2}$. Solid and dashed lines indicate the result of the linear fit and its one sigma error. The labeled numbers in the figure correspond to the name of the objects that are used in this analysis as follows; 1. Mon R2 IRS3, 2. Orion IRC2, 3. S140, 4. Elias 29, 5. AFGL 2136, 6. W33A, 7. NGC 7538 IRS9.

\section{References}

Aguirre, J. E., Bezaire, J. J., Cheng, E. S., et al. 2003, ApJ, 596, 273 Alves, D. R. 2004, New Astron. Rev., 48, 659

Bernstein, M. P., Sandford, S. A., \& Allamandola, L. J. 2000, ApJ, 542, 894

Boogert, A. C. A., \& Ehrenfreund, P. 2004, in Astrophysics of Dust, ed. A. N. Witt, G. C. Clayton, \& B. T. Draine, ASP Conf. Ser., 309, 547

Boogert, A. C. A., Ehrenfreund, P., Gerakines, P. A., et al. 2000, A\&A, 353, 349 Boogert, A. C. A., Pontoppidan, K. M., Knez, C., et al. 2008, ApJ, 678, 985

Chiar, J. E., Gerakines, P. A., Whittet, D. C. B., et al. 1998, ApJ, 498, 716

Dartois, E., Schutte, W., Geballe, T. R., et al. 1999, A\&A, 342, L32

D’Hendecourt, L. B., \& Allamandola, L. J. 1986, A\&AS, 64, 453

Ehrenfreund, P., \& Schutte, W. A. 2000, Adv. Space Res., 25, 2177

Ehrenfreund, P., Boogert, A. C. A., Gerakines, P. A., et al. 1996, A\&A, 315, L341

Gerakines, P. A., Schutte, W. A., Greenberg, J. M., \& van Dishoeck, E. F. 1995, A\&A, 296, 810

Gerakines, P. A., Schutte, W. A., \& Ehrenfreund, P. 1996, A\&A, 312, 289

Gerakines, P. A., Whittet, D. C. B., Ehrenfreund, P., et al. 1999, ApJ, 522, 357

Gibb, E. L., Whittet, D. C. B., Schutte, W. A., et al. 2000, ApJ, 536, 347

Gibb, E. L., Whittet, D. C. B., Boogert, A. C. A., \& Tielens, A. G. G. M. 2004, ApJS, 151, 35

Gruendl, R. A., \& Chu, Y. 2009, ApJS, 184, 172

Houck, J. R., Roellig, T. L., van Cleve, J., et al. 2004, ApJS, 154, 18

Israel, F. P., de Graauw, T., van de Stadt, H., \& de Vries, C. P. 1986, ApJ, 303, 186

Ita, Y., Onaka, T., Kato, D., et al. 2008, PASJ, 60, 435

Kato, D., Nagashima, C., Nagayama, T., et al. 2007, PASJ, 59, 615

Lee, D., Pak, S., Dixon, W. V. D., \& van Dishoeck, E. F. 2007, ApJ, 655, 940

Luck, R. E., Moffett, T. J., Barnes, III, T. G., \& Gieren, W. P. 1998, AJ, 115, 605

Meixner, M., Gordon, K. D., Indebetouw, R., et al. 2006, AJ, 132, 2268

Millar, T. J., Bennett, A., Rawlings, J. M. C., Brown, P. D., \& Charnley, S. B. 1991, A\&AS, 87, 585

Murakami, H., Baba, H., Barthel, P., et al. 2007, PASJ, 59, 369

Nummelin, A., Whittet, D. C. B., Gibb, E. L., Gerakines, P. A., \& Chiar, J. E. 2001, ApJ, 558, 185

Öberg, K. I., Fraser, H. J., Boogert, A. C. A., et al. 2007, A\&A, 462, 1187

Ohyama, Y., Onaka, T., Matsuhara, H., et al. 2007, PASJ, 59, 411
Oliveira, J. M., van Loon, J. T., Chen, C., et al. 2009, ApJ, 707, 1269

Onaka, T., Matsuhara, H., Wada, T., et al. 2007, PASJ, 59, 401

Pendleton, Y. J., Tielens, A. G. G. M., Tokunaga, A. T., \& Bernstein, M. P. 1999, ApJ, 513, 294

Pontoppidan, K. M., Fraser, H. J., Dartois, E., et al. 2003, A\&A, 408, 981

Pontoppidan, K. M., Boogert, A. C. A., Fraser, H. J., et al. 2008, ApJ, 678, 1005

Robitaille, T. P., Whitney, B. A., Indebetouw, R., \& Wood, K. 2007, ApJS, 169 , 328

Ruffle, D. P., \& Herbst, E. 2001, MNRAS, 324, 1054

Sakon, I., Onaka, T., Kaneda, H., et al. 2006, ApJ, 651, 174

Schutte, W. A., \& Greenberg, J. M. 1997, A\&A, 317, L43

Seale, J. P., Looney, L. W., Chu, Y., et al. 2009, ApJ, 699, 150

Shimonishi, T., Onaka, T., Kato, D., et al. 2008, ApJ, 686, L99

Shore, S. N. 2002, The Tapestry of Modern Astrophysics, ed. S. N. Shore, 201, 478

Sloan, G. C., Kraemer, K. E., Price, S. D., \& Shipman, R. F. 2003, ApJS, 147, 379

Spoon, H. W. W., Moorwood, A. F. M., Pontoppidan, K. M., et al. 2003, A\&A, 402, 499

Thi, W., van Dishoeck, E. F., Dartois, E., et al. 2006, A\&A, 449, 251

Tielens, A. G. G. M. 2005, The Physics and Chemistry of the Interstellar Medium van den Ancker, M. E., Tielens, A. G. G. M., \& Wesselius, P. R. 2000, A\&A, 358,1035

van der Marel, R. P. \& Cioni, M. 2001, AJ, 122, 1807

van Dishoeck, E. F., \& Blake, G. A. 1998, ARA\&A, 36, 317

van Loon, J. T., Oliveira, J. M., Wood, P. R., et al. 2005, MNRAS, 364, L71

van Loon, J. T., Oliveira, J. M., Gordon, K. D., et al. 2010a, AJ, 139, 68

van Loon, J. T., Oliveira, J. M., Gordon, K. D., Sloan, G. C., \& Engelbracht, C. W. 2010b, AJ, 139, 1553

Watanabe, N., Mouri, O., Nagaoka, A., et al. 2007, ApJ, 668, 1001 Werner, M. W., Roellig, T. L., Low, F. J., et al. 2004, ApJS, 154, 1 Whitney, B. A., Sewilo, M., Indebetouw, R., et al. 2008, AJ, 136, 18

Whittet, D. C. B., Gerakines, P. A., Tielens, A. G. G. M., et al. 1998, ApJ, 498, L159

Whittet, D. C. B., Shenoy, S. S., Bergin, E. A., et al. 2007, ApJ, 655, 332

Zaritsky, D., Harris, J., Thompson, I. B., \& Grebel, E. K. 2004, AJ, 128, 1606

Zasowski, G., Kemper, F., Watson, D. M., et al. 2009, ApJ, 694, 459 